\title{
Efecto del Phlebodium Decumanum sobre los cambios en niveles plasmáticos de testosterona y cortisol inducidos por el ejercicio en sujetos no entrenados
}

\author{
José A Gonzalezjurado ${ }^{1 a}$, Carlos de Teresa $\mathbf{G}^{2}$, Edgardo Molina $\mathbf{S}^{3 \mathrm{~b}}$, \\ Francisco Pradas de la $\mathrm{F}^{4 a}$, Rafael Guisado $\mathrm{B}^{5}$, José $\mathrm{N}$ aranjo $0^{6}$. \\ Effects of the consumption of Phlebodium \\ Decumanum on plasma cortisol and \\ testosterone levels in subjects \\ participating in an exercise program
}

\begin{abstract}
Background: Phlebodium Decumanum is a type of fern that could have an immune or stress response modulating action. Aim: To evaluate if the consumption of a preparation obtained from Phlebodium Decumanum, modifies the changes induced by exercise in plasma levels of testosterone and cortisol. Material and methods: Fourteen male subjects aged $22 \pm 2$ years were randomized to consume Phlebodium Decumanum and 10 subjects, aged $23 \pm 1$ years were ascribed to consume a placebo. Both groups performed a standard exercise program, three times a week during one month. Plasma free testosterone and cortisol levels were measured before and 48 hours after the last session of the exercise program. Results: Cortisol levels increased significantly from $18.9 \pm 6.8$ to $26.2 \pm 5.9 \mu \mathrm{g} / \mathrm{dl}$, in the control group. In the group consuming the study product, no changes were detected $(20.0 \pm 4.6$ and $20.9 \pm 5.9) \mu \mathrm{g} / \mathrm{dl}$ before and after the exercise program, respectively). Plasma free testosterone decreased significantly in both groups from $36.1 \pm 11.04$ to 20.7 $\pm 4.3 \mathrm{pg} / \mathrm{ml}$ in the control group and from $33.7 \pm 10.0 \mathrm{pg} / \mathrm{ml}$ to $18.6 \pm 4.9 \mathrm{pg} / \mathrm{ml}$ in study group. Conclusions: The cortisol levels increased significantly in the control group but not in the study group. Therefore we can suggest that consumption of Phlebodium Decumanum would be able to modulate the response of this hormone to physical stress (Rev Méd Chile 2009; 137: 497-503).
\end{abstract}

(Key words: Excercise tests; Ferns; Hydrocortisone)

Recibido el 24 de abril, 2008. Aceptado el 9 de enero, 2009.
${ }^{1}$ Facultad de Ciencias del Deporte, Universidad Pablo de Olavide de Sevilla. España. ${ }^{2}$ Centro
Andaluz de Medicina del Deporte. Granada. España. ${ }^{3}$ Departamento de Educación Física, De-
portes y Recreación, Universidad Metropolitana de Ciencias de la Educación. Santiago, Chile.
${ }^{4}$ Facultad de Ciencias de la Salud y el Deporte. Universidad de Zaragoza. ${ }^{5}$ Escuela Ciencias de
la Salud, Universidad de Granada. ${ }^{6}$ Centro Andaluz de Medicina del Deporte. Sevilla, España.
aDoctor en Actividad Física y Salud por la Universidad de Granada. Licenciado en Cien-
cias de la Actividad Física y el Deporte. Universidad de Granada.
bDoctor en Actividad Física y Salud por la Universidad de Granada. Licenciado en Educa-
ción Física, Universidad de Chile.

Correspondencia a: José Antonio Gonzalezjurado: C/ Gradeo, 12. Castilleja de la Cuesta, C.P. 41950. Sevilla. España. Fax: 00-34-954348377. E mail: jagonjur@upo.es 
Los os deportistas siempre han buscado recursos y ayudas que les permitan mejorar y optimizar su rendimiento deportivo. A este tipo de productos se les ha llamado ayudas ergogénicas. Este término actualmente es más amplio, y abarca la utilización de cualquier elemento que tenga como objetivo obtener un mejor rendimiento deportivo o una limitación de las consecuencias negativas del entrenamiento o la competición. Se trata por tanto de agentes farmacológicos, agentes hormonales, agentes fisiológicos, agentes y sustancias nutricionales, fenómenos psicológicos y factores mecánicos ${ }^{1}$.

Varias hormonas, neurotransmisores y moléculas metabólicamente activas son modificadas por el ejercicio físico. Entre estas sustancias se encuentran la testosterona y el cortisol, consideradas hormonas de respuesta al estrés. Los cambios en sus niveles circulantes se relacionan con niveles de rendimiento físico y se consideran indicadores de situaciones de sobrecarga física e incluso de sobreentrenamiento ${ }^{2-11}$. El cortisol es una hormona íntimamente relacionada con el estrés y los procesos catabólicos. Podemos afirmar que la respuesta más frecuentemente informada respecto a las concentraciones plasmáticas de cortisol, consiste en que tras la práctica física intensa durante periodos de tiempo de corta o media duración, se suele producir un aumento de los niveles sanguíneos de cortisol, junto con otros glucocorticoides y se puede considerar como un índice de sobresolicitación y overreaching ${ }^{12,13}$.

La testosterona es la principal hormona esteroidea anabolizante y andrógenica en el hombre, ejerce numerosos efectos en los procesos metabólicos fisiológicos. Además con su acción contrarresta el efecto provocado por el cortisol durante la práctica de ejercicio físico intenso ${ }^{14}$. Muchos estudios, informan de una disminución de la testosterona cuando sometemos al sujeto a un estrés físico al cual su estado de forma física no está capacitado para responder ${ }^{15,16}$.

Se ha informado que el ejercicio físico intenso provoca un incremento de los procesos catabólicos, con disminución de testosterona libre, índice testosterona libre/cortisol e incremento de cortisol, mientras que en el caso de utilización de cargas ligeras, conlleva un incremento de testosterona libre, índice testosterona/cortisol y disminución del cortisol $^{3,4,7,17}$.
Situaciones de sobrecarga o fatiga subaguda (overreaching) se caracterizan por una disminución significativa y constante de los niveles de testosterona, normalmente asociada a una elevación de los niveles de cortisol plasmático. La relación entre la testosterona libre y el cortisol se ha sugerido como indicador de una aproximación al estado anabólico/catabólico de un individuo. Incrementos del estrés provocado por el ejercicio intenso se han relacionado con disminuciones en el índice testosterona libre/cortisol $2,8,17$.

El Phlebodium decumanum es un helecho de la familia de las polipodiáceas. Estos helechos son cultivados en monocultivo en la plantación del lago Yojoa en Honduras. Se caracteriza por un amplio fronde provisto de varios soros (3 a 7) y por su grueso, carnoso y velloso rizoma.

Los efectos de los derivados de este helecho están relacionados con la modulación de la respuesta inmunológica como consecuencia de situaciones de estrés, tal y como se demuestra en el estudio in vitro ${ }^{18}$, así como in vivo en humanos ${ }^{19-22}$.

La acción protectora del Phlebodium decumanum en modelos experimentales in vitro, ha sido estudiada por Punzón et al (2003) ${ }^{18}$ en el Centro de Biología Molecular "Severo Ochoa" (Universidad Autónoma de Madrid). El perfil modulador de las respuestas al estrés, lo hace potencialmente atractivo para prevenir los efectos negativos de la sobresolicitación muscular, que puede estar relacionado con el desajuste de la respuesta hormonal y el desequilibrio anabólico/catabólico.

El objetivo de este estudio es comprobar si el aporte nutricional de Phlebodium decumanum, tiene efectos sobre los cambios en los niveles plasmáticos inducidos por el ejercicio en hormonas indicadoras de estrés fisiológico como la testosterona y cortisol.

\section{MATERIAL Y MÉTODO}

A partir de una muestra de 24 varones (estudiantes universitarios), se conformaron dos grupos distribuidos aleatoriamente según el consumo máximo de oxígeno determinado mediante protocolo en tapiz rodante (modelo Runrace D-140 de Technogym). Se utilizó el analizador de gases Oxicon Delta de Jaeger. 
Un grupo de 14 sujetos consumió Phlebodium decumanum (G.PD), $(22,14 \pm 1,56$ años, peso $75,05 \pm 8,92 \mathrm{~kg}$ ) y un segundo grupo de 10 sujetos se les administró un placebo (G.P.) $(22,8 \pm 1,22$ años; peso $82,78 \pm 9,82.5 \mathrm{~kg}$ ).

Se sometieron a un programa de ejercicio de un mes de duración. Todos los sujetos firmaron un documento de consentimiento informado, sobre las características del producto, el placebo y el tipo de entrenamiento a realizar, siguiendo las indicaciones de la Declaración de Helsinki.

Las variables independientes con las que se trabajó fueron: En primer lugar el consumo de producto o de placebo. La formulación utilizada a base de Phlebodium decumanum se obtienen a partir de una fracción hidrosoluble de fronda purificada y estandarizada. Esta fracción se obtiene por extracción hidroalcohólica de las frondas maduras, secas y trituradas, seguida de la eliminación del disolvente orgánico, concentración de la fase acuosa y purificación. La mezcla de extracto con rizoma esterilizado y triturado, seguida de secado y homogeneización, da lugar a un polvo, que puede ser administrado en diversas presentaciones farmacéuticas.

Para nuestro estudio elegimos la forma encapsulada por su mayor comodidad de administración. El método de producción del extracto está protegido bajo patente (P-9900133 ${ }^{\circledR}$ ).

El preparado consistió en cápsulas de $400 \mathrm{mg}$, cuyo contenido es el siguiente: $250 \mathrm{mg}$ de extracto de fracción hidrosoluble del fronde y $150 \mathrm{mg}$ de polvo de rizoma de Phlebodium decumanum.

El placebo consistió en las mismas cápsulas pero con $400 \mathrm{mg}$ de levadura de cerveza. Tanto el producto estudiado como el placebo fueron suministrados por la empresa Helsint S.A.L. La dosificación del producto y el placebo fue 2 cápsulas/ 3 veces al día.

La segunda variable independiente fue el programa de ejercicio de tres sesiones semanales, realizado por toda la muestra, que consistió:

1‥ Golpeos de tenis en cancha. Trabajando por parejas, cada sujeto ejecutó 500 golpeos; golpe de derecha paralelo: 125, golpe de derecha cruzado: 125; revés paralelo: 125; revés cruzado: 125 .

2o. Trabajo de fuerza dinámica, por tres grupos musculares: Pectorales: press de banca. Dorsales: Lat tras nuca (jalones tras nuca). Deltoides: press sentado con mancuernas (seated dumbdell press).

Se trabajó a una intensidad de $55 \%$ y $60 \%$, del test máximo, hasta la fatiga en cada serie. La fuerza máxima dinámica se determinó mediante el test propuesto por Brzycki citado por García $(1996)^{23}$. Se realizaron tres series de cada ejercicio las dos primeras semanas y cuatro series la tercera y cuarta semana. La recuperación entre series fue de 2 minutos.

3o. Entrenamiento de resistencia mediante un interval-training. En cada serie se realizó 10 veces un recorrido de ida y vuelta a máxima intensidad sobre una distancia de 8 metros, completándose por tanto 160 metros por serie. La pausa venía determinada por la frecuencia cardiaca (FC), así se inicia la siguiente serie cuando la FC está entre 125-130 lat: $\mathrm{min}^{-1}$, registrada mediante un pulsómetro de muñeca Polar Vantage NV. Se realiza este trabajo durante 20 min las dos primeras semanas, durante 25 min la tercera y cuarta semana y durante 30 min la primera y la última sesión.

Las variables dependientes analizadas en este estudio fueron: niveles plasmáticos de cortisol y de testosterona libre, medidos en sangre venosa periférica.

Se tomaron muestras antes de iniciar el protocolo y $48 \mathrm{~h}$ después de la última sesión de trabajo. Todas las extracciones de sangre venosa fueron realizadas por diplomados en Enfermería con experiencia contrastada.

Las mediciones de cortisol y testosterona total se realizaron a través de técnicas de inmunoquimioluminiscencia. La máquina utilizada fue $\mathrm{Ni}$ chols Advantage ${ }^{\circledR}$.

La testosterona libre se analizó mediante enzimainmunoanálisis. El aparato usado fue el modelo Vidas de la casa Byo-Merieux ${ }^{\circledR}$.

En cuanto al diseño de esta investigación, se trata en un estudio a doble ciego, multigrupo con dos grupos aleatorios; grupo experimental (consume Phlebodium decumanum) y grupo de control (consume placebo). Se han llevado a cabo medidas al comienzo y al final del protocolo.

El tratamiento estadístico de los datos se efectuó con el software SPSS14.0, y fue el siguiente: en primer lugar se han realizado pruebas de valoración de la normalidad (test de Shapiro-Wilk)

ARtíCULO DE 
para cada variable, tanto para contrastes intragrupos como para contrastes entre grupos. $\mathrm{Si}$ la variable a analizar cumplía la condición de normalidad, tanto para contrastar los cambios de variables intragrupos (datos apareados) como para intergrupos (muestras independientes), se utilizó la prueba t de Student.

Si la variable estudiada no cumplía la normalidad, se efectuaron pruebas de contraste no paramétricas. Para contrastar los cambios de variables intragrupos se efectuó la prueba de Wilcoxon, para contrastes de hipótesis de dos muestras intergrupos se hizo la prueba U de Mann-Whitney.

Se consideró significativo un valor de $\mathrm{p}$ menor de 0,05 .

\section{RESULTADOS}

En la Tabla 1 se presenta los valores de los estadísticos descriptivos obtenidos en cada una de las variables dependientes evaluadas en este estudio.

En la Figura 1 se presenta las medias de los niveles de testosterona libre $(\mathrm{pg} / \mathrm{ml})$ en los dos grupos, comparándose pre-test y post-test. En ambos grupos se observa una notable disminución de los niveles de esta hormona con diferencias significativas en el grupo placebo p <0,001 y para el grupo PD p <0,0001 (prueba t de Student). En la Figura 2 se muestran las diferencias entre el pre y post-test en los niveles plasmáticos de cortisol ( $\mu \mathrm{g} / \mathrm{dl})$, obteniéndose un aumento estadís-

Tabla 1. Estadísticos descriptivos de las variables dependientes

\begin{tabular}{|llcllrl|}
\hline & \multicolumn{3}{c}{$\begin{array}{c}\text { Grupo PD } \\
\text { Media }\end{array}$} & D.S. & $\mathrm{n}$ & Mrupo placebo \\
& $\mathrm{n}$ & & & & & D.S. \\
\hline Testosterona libre pret. & 14 & 33,66 & 10,01 & 10 & 36,10 & 11,04 \\
Testosterona libre post & 14 & 18,61 & 4,93 & 10 & 20,72 & 4,3 \\
\% cambio test. libre & 14 & $-39,92$ & 23,38 & 10 & $-39,63$ & 15,66 \\
Cortisol pre-test & 14 & 20,04 & 4,59 & 10 & 18,89 & 6,83 \\
Cortisol post-test & 14 & 20,95 & 5,92 & 10 & 26,19 & 5,93 \\
\% cambio cortisol & 14 & 6,35 & 28,35 & 10 & 54,93 & 68,41 \\
İndice test./cort. pret. & 14 & 1,71 & 0,52 & 10 & 2,25 & 1,34 \\
Índice test/cort post & 14 & 0,96 & 0,4 & 10 & 0,82 & 0,2 \\
\% cambio índice t/c & 14 & $-38,41$ & 33,09 & 10 & $-53,14$ & 25,28 \\
\hline
\end{tabular}

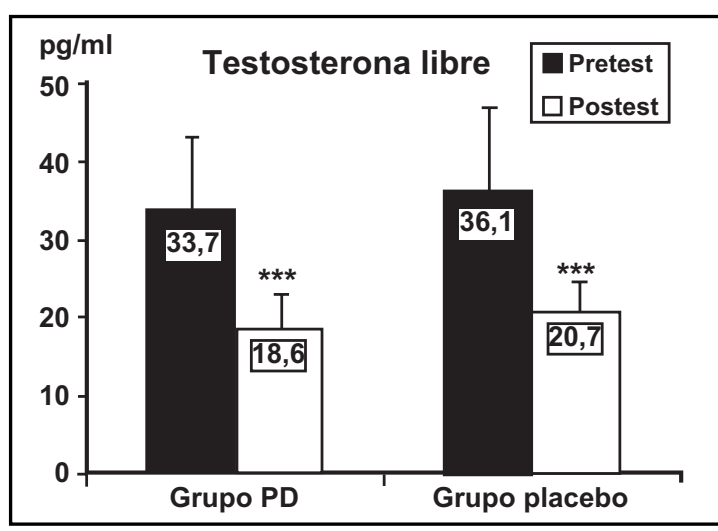

Figura 1. Comparación intragrupo de medias pre y postest

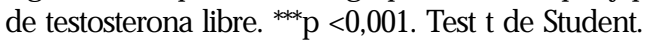

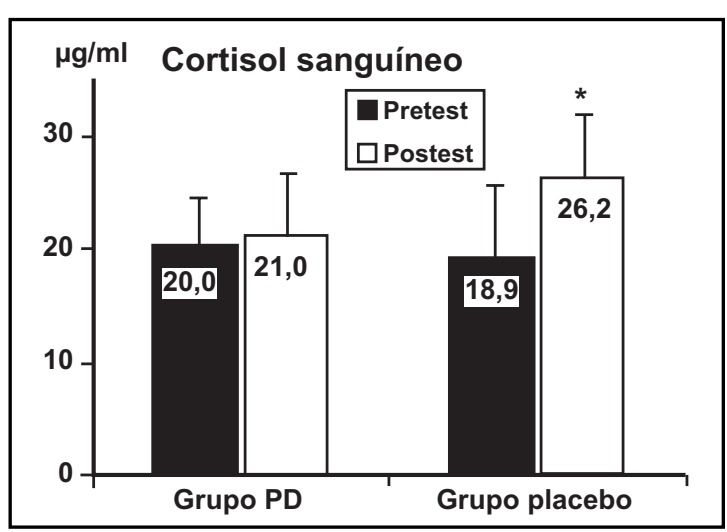

Figura 2. Comparación intragrupo de medias pre y postest de cortisol en sangre. *p $\triangleleft 0.05$. Test t de Student. 
ticamente significativo (t de Student) en el grupo placebo, $p<0,05$, mientras que en el grupo PD prácticamente no se observan cambios entre pre y post-test. En la Figura 3 se puede ver que el índice testosterona libre/cortisol sufre una disminución claramente significativa en ambos grupos; $\mathrm{p}<0,01$, resultados obtenidos mediante la prueba de Wilcoxon. En la Figura 4 se presenta los resultados obtenidos en el porcentaje de cambio, así podremos analizar la mejora (o disminución) relativa, producida en cada grupo y a partir de ahí comparar la magnitud de dicha variación entre uno y otro grupo. No detectamos diferencias significativas intergrupos en dos de las tres variables evaluadas. El porcentaje de cambio de cortisol en sangre, sí que es significativamente distinto

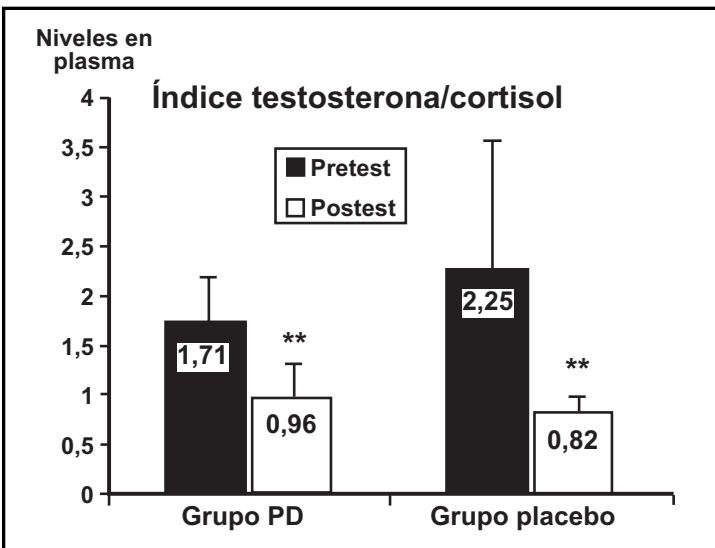

Figura 3. Comparación intragnupo de medias pre y postest de índice testosterona/cortisol en sangre. * ${ }^{*} \varangle, 01$. entre grupos, así en el grupo $\mathrm{P}$ los niveles plasmáticos de esta hormona catabólica aumentan significativamente más que el grupo PD ( $p<0,05$; Mann-Whitney).

\section{Discusión}

De las determinaciones hormonales realizadas en nuestro trabajo, ha sido el cortisol el que ha amojado resultados más destacados. Así, en el grupo placebo, hemos obtenido resultados acordes con la mayoná de la documentación revisada, es decir, hemos detectado aumentos significativos entre el pre-test y el post-test ( $p<0,01)$. Similares resultados se muestran en un estudio realizado por Viru et al (2001) $)^{11}$, donde informan que tras la realización de un trabajo físico a $70 \%$ del consumo máximo de oxígeno, obtuvieron un aumento en la concentración de cortisol, manteniéndose estable la testosterona en todos los sujetos (n $=12$ ). Otros estudios informan asimismo de un aumento de los niveles plasmáticos de cortisol durante el ejercicio, además, tanto el incremento como el retorno a niveles basales dependen de la intensidad y la duración del ejercicio ${ }^{24-26}$. Tanto en el ejercicio intenso como moderado, el cortisol permanece elevado hasta aproximadamente $90 \mathrm{~min}$ después del término del ejercicio ${ }^{27}$.

Sin embargo, el grupo PD en lugar de aumentar el cortisol plasmático se ha mantenido sin cambios estadísticamente significativos. Podemos inferir, por tanto, que el consumo de Phlebodium decumanum protege al sujeto del estrés causado por el ejercicio físico. En este sentido la comparación intergrupos

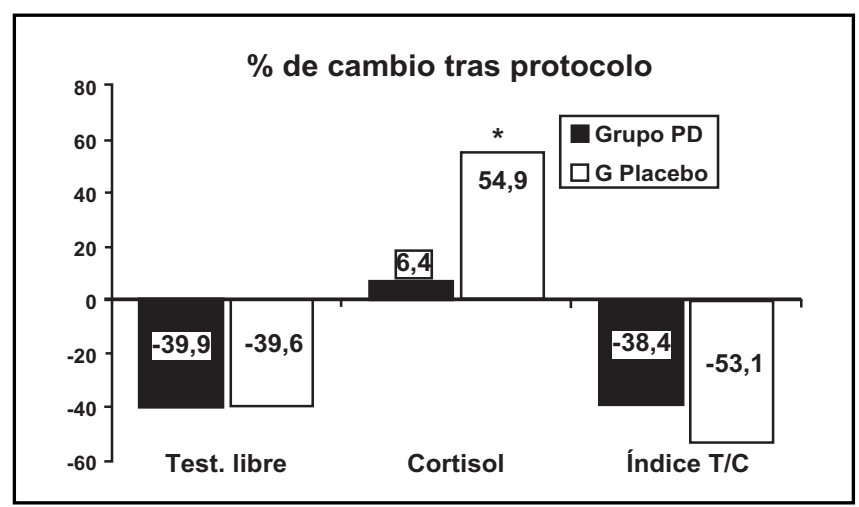

Figura 4. Comparación intragrupo de medias de porcentaje de cambio en hormonas e índice testosterona/ cortisol en plasma.

Artículo de 
también proporciona diferencias significativas respecto a esta hormona, en el porcentaje de cambio al inicio y al final del entrenamiento ( $p<0,05$ ).

En algunas publicaciones se ha informado que el cortisol sufre una disminución de los niveles plasmáticos en situaciones de exceso de trabajo físico, pero esta situación sucede en los estados de síndrome de sobreentrenamiento cronificado 7,10-13,28-30

Respecto a la testosterona encontramos algunas publicaciones que informan acerca de un aumento o de la inexistencia de cambios en los niveles plasmáticos de dicha hormona con el ejercicio físico sistematizado de cierta intensidad ${ }^{6,31}$. Sin embargo, podemos observar que se refiere a situaciones en que a sujetos con un alto nivel de entrenamiento se aplicó un estímulo físico de una sesión única, por lo que se trata de una respuesta aguda al estrés. La mayoría de los estudios informan que cuando el estrés físico es de cierta intensidad y se mantiene por un tiempo relativamente prolongado, los niveles plasmáticos de la testosterona, la testosterona libre y el índice testosterona cortisol disminuyen significativamente $^{8,32-34}$.

Los resultados que hemos obtenido referentes a estas variables coinciden con lo reportado en la documentación anteriormente citada. Se registró una considerable disminución de los niveles de testosterona libre en ambos grupos (grupo PD: $p>0,001$ y grupo placebo: $p<0,001$ ). Como consecuencia de este importante descenso en los niveles de testosterona libre, la relación testosterona/cortisol también sufrió decrementos que fueron estadísticamente significativos en ambos grupos (grupo PD y grupo placebo: $\mathrm{p}<0,01$ ). Por consiguiente, si nos atenemos a la interpretación de estos valores podemos afirmar que la muestra se sometió a un trabajo físico que supuso un estrés considerable en función de su condición física previa.

\section{REFERENCIAS}

1. Banfi G, Marinem M, Roi GS, Agape V. Usefulness of free testosterone/cortisol ratio during a season of elite speed skating athletes. Int J Sports Med 1993; 14: 373-9.

2. Hoogeveen AR, ZonderLand ML. Relationships between testosterone, cortisol and performance in professional cyclists. Int J Sports Med 1996; 17: 423-8.

3. HÄKKINEN K, PAKARINEN A. Senum hormones in male strength athletes during intensive short tem strength training. EurJ Appl Physiol Occup Physiol 1991; 63: 194-9.
Otro trabajo que aporta conclusiones en esta línea es el de Urhausen et al (1995) ${ }^{13}$, en el que la relación. testosterona/cortisol disminuye en relación con la intensidad y la duración del ejercicio, así como durante periodos de entrenamiento intenso o de competiciones frecuentes. Esta disminución puede ser revertida con medidas regenadoras. La evolución de esta variable y su interpretación en relación a la valoración del entrenamiento aún presenta muchas incógnitas si atendemos a las investigaciones realizadas. En cualquier caso, la mayońa de los estudios no han sido capaces de confirmar cambios en la relación testosterona/cortisol en deportistas de fuerza o de resistencia sobreentrenados ${ }^{17,35,36}$. Los resultados obtenidos sugieren por tanto que el protocolo de ejercicios ejecutado por nuestra muestra fue lo suficientemente intenso para provocar evidentes signos de estrés físico, reflejados en la disminución de testosterona libre, e índice testosterona cortisol, sin embargo, no fue lo bastante exigente en duración o intensidad como para llevar a los deportistas a un estado de sobreentrenamiento. Aunque tal vez sí podríamos hablar de una situación de fatiga subaguda o sobresolicitación (overreaching).

Asimismo, estos resultados apuntan a afirmar que los sujetos que suplementaron su alimentación con Phlebodium decumanum manifestaron menor grado de estrés físico, tal y como refleja su respuesta referida a las concentraciones de cortisol. Por consiguiente soportaron mejor el trabajo al que fueron sometidos y estanian potencialmente mejor preparados para soportar mayores cargas de entrenamiento.

Agradecimientos

En este estudio ha colaborado la empresa Helsint S.A.L, sin cuya colaboración no se podría haber llevado a cabo el mismo.

4. Jacks DE, Sowash J, Anning J, McGloughlin T, Andres F. Effect of exercise at three exercise intensities on salivary cortisol. J Strength Cond Res 2002; 16: 286-9.

5. JÜRIMÄE J, JÜrImÄE T, PuRge P. Plasma testosterone and cortisol responses to prolonged sculling in male competitive rowers. J Sports Sci 2001; 19: 893-8.

6. Kraemer WJ, Fleck SJ, Cawister R, Shealy M, Dudiey GA, MARESH CM ET AL. Training responses of plasma beta-endorphin, adrenocorticotropin, and cortisol. Med Sci Sports Exerc 1989; 21: 146-53. 
7. Marinelu M, Roi GS, Giacometti M, Bonini P, Banfi G. Cortisol, testosterone, and free testosterone in athletes performing a marathon at 4,000 m altitude. Horm Res 1994; 41: 225-9.

8. RAASTAD T, BJøRO T, HALÉN J. Hormonal responses to high- and moderate-intensity strength exercise. Eur J Appl Physiol 2000; 82: 121-8.

9. URHAUSEN A, KINDERMANN W. Diagnosis of overtraining: what tools do we have? Sports Med 2002; 32: 95-102.

10. Viru AM, HackNey AC, VäLja E, Karelson K, Janson T, VIRU M. Influence of prolonged continuous exercise on hormone responses to subsequent exercise in humans. Eur J Appl Physiol 2001; 85: 578-85.

11. Punzón C, Alcaide A, Fresno M. In vitro anti-inflammatory activity of Phlebodium decumanum. Modulation of tumor necrosis factor and soluble TNF receptors. Int Immunopharmacol 2003; 3: 1293-9.

12. Tuominen M, Bohun L, Rolfsen W. Effects of Calaguala and an active principle, adenosine, on platelet activating factor. Planta Med 1992; 58: 306-10.

13. Vasange M, Lu B, Welch CJ, Rolfsen W, Bohun L. The flavonoid constituents of two Polypodium species (Calaguala) and their effect on the elastase release in human neutrophils. Planta Med 1997; 63: 511-7.

14. Vasange M, RolfSen W, Bohuin L A sulphonoglycolipid from the fern Polypodium decumanum and its effect on the platelet activating-factor receptor in human neutrophils. J Pharm Pharmacol 1997; 49: 562-6.

15. Vasange-Tuominen M, Perera-Ivarsson P, Shen J, Bohin L, Rolfsen W. The fern Polypodium decumanum, used in the treatment of psoriasis, and its fatty acid constituents as inhibitors of leukotriene B4 formation. Prostaglandins Leukot Essent Fatty Acids 1994; 50: 279-84.

16. Ganong WF. Fisiología Médica. Mexico D.F.: Editorial El Manual Moderno. 1990; 318-82.

17. GLEESON M. Biochemical and immunological markers of overtraining. Journ Sport Sci Med 2002; 2: 31-41.

18. Urhausen A, Gabriel H, Kindermann W. Blood hormones as markers of training stress and overtraining. Sports Med 1995; 20: 251-76.

19. Campusano C, Brusco F, Campino C, Rodríguez L, ARteaga E. Comparación de distintos métodos para evaluar la función androgénica en el adulto mayor. Rev Méd Chile 2006; 134: 1123-8.

20. Guezennec CY, Lafarge JP, Bricout VA, Merino D, SERRURIER B. Effect of competition stress on tests used to assess testosterone administration in athletes. Int J Sports Med 1995; 16: 368-72.

21. Consitt LA, Copeiand JL, Trembiay MS. Endogenous anabolic hormone responses to endurance versus resistance exercise and training in women. Sports Med 2002; 32: 1-22.

22. SLOWinSKa-LisowsKa M, MajDA J. Hormone plasma levels from pituitary-gonadal axis in performance athletes after the $400 \mathrm{~m}$ nu. J Sports Med Phys Fitness 2002; 42: 243-9.

23. GarCía JM, NAVARRO M, Rúz JA. Bases teóricas del entrenamiento deportivo. Madrid: Editorial Gymnos. 1996; 170.
24. Viru A, Viru M, Karelson K, Janson T, Sim K, Fischer $\mathrm{K}$, HACKNEY AC. Adrenergic effects on adrenocortical cortisol response to incremental exercise to exhaustion. Eur J Appl Physiol 2007; 100: 241-5.

25. Brenner I, Shek PN, Zamecnik J, Shephard RJ. Stress hormones and the immunological responses to heat and exercise. Int J Sports Med 1998; 19: 130-43.

26. Barron JL, Noakes TD, Levy W, Smith C, Milar RP. Hypothalamic dysfunction in overtrained athletes. J Clin Endocrinol Metab 1985; 60: 803-6.

27. Woods JA. Exercise and neuroendocrine modulation of macrophage function. Int Journ Sport Med 2000; 21: 24-30.

28. Lehmann M, Gastmann U, Petersen KG, Bachl N, Seidel A, KhalaF AN, Fischer S, KeUl J. Training-overtraining: performance, and hormone levels, after a defined increase in training volume versus intensity in experienced middle- and long-distance runners. Br J Sports Med 1992; 26: 233-42.

29. Kirwan JP, Costill DL, Flynn MG, Mitchell JB, Fink WJ, Neufer PD, Houmard JA. Physiological responses to successive days of intense training in competitive swimmers. Med Sci Sports Exerc 1988; 20: 255-9.

30. Fry AC, Kraemer WJ, Ramsey LT. Pituitary-adrenalgonadal responses to high-intensity resistance exercise overtraining. J Appl Physiol 1998; 85: 2352-9.

31. FAHRNER CL, HACKNEY AC. Effects of endurance exercise on free testosterone concentration and the binding affinity of sex hormone binding globulin (SHBG). Int J Sports Med 1998; 19: 12-5.

32. Schmid P, Pusch HH, Wolf W, Pilger E, Pessenhofer H, SCHWABERGER G ET AL. Serum FSH, LH, and testosterone in humans after physical exercise. Int J Sports Med 1982; 3: 84-9.

33. Bosco C, Cow R, Bonomi R, Von Duvilard SP, Viru A. Monitoring strength training: neuromuscular and hormonal profile. Med Sci Sports Exerc 2000; 32: 202-8

34. Wheeier GD, Singh M, Pierce WD, Eping WF, Cumming DC. Endurance training decreases senum testosterone levels in men without change in luteinizing homone pulsatile release. J Clin Endocrinol Metab 1991; 72: 422-5

35. Houmard JA, Costill DL, Mitchel JB, Park SH, Fink WJ, BurNS JM. Testosterone, cortisol, and creatine kinase levels in male distance runners during reduced training. Int J Sports Med 1990; 11: 41-5.

36. BANFI G, DolcI A. Free testosterone/cortisol ratio in soccer: usefulness of a categorization of values. J Sports Med Phys Fitness 2006; 46: 611-6.

37. LAC G, BerThon P. Changes in cortisol and testosterone levels and $\mathrm{T} / \mathrm{C}$ ratio during an endurance competition and recovery. J Sports Med Phys Fitness 2000; 40: 139-44.

38. Mackinnon LT, Hooper SL, Jones S, Gordon RD, BACHMANN AW. Hormonal, immunological, and hematological responses to intensified training in elite swimmers. Med Sci Sports Exerc 1997; 29: 1637-45.

Artículo de 